\title{
Acupuncture for cosmetic use: a systematic review of prospective studies
}

\author{
Byung-Cheul Shin, KMD, PhD ${ }^{1,2}$, Kyeong-Tae Lim, KMD² \\ ${ }^{1}$ Division of Clinical Medicine, School of Korean Medicine, Pusan National University, Yangsan, Rep. of Korea, ${ }^{2}$ Department of Korean \\ Rehabilitation Medicine, Pusan National University Korean Medicine Hospital, Yangsan, Rep. of Korea
}

\begin{abstract}
Background: Despite the use of cosmetic acupuncture in clinical fields, there is little evidence regarding its effects and safety for cosmetic use.

Objective: Objectives of this article are to review the literature and systematically evaluate the effects and current use of acupuncture for cosmetic purposes.

Methods: We searched 15 English, Chinese, Japanese, and Korean databases by using relevant keywords. All prospective studies on acupuncture for cosmetic use were considered; we conducted literature analyses to determine the current status of cosmetic acupuncture use and extracted data related to its effects.

Results: Two randomized controlled trials and 5 single-armed prospective trials with 216 participants ( 150 from randomized controlled trials, 66 from single-armed studies) were included. All 7 studies reported positive results for at least one of the following: total effective rate $(n=2)$, squares and counts of wrinkles $(n=1)$, facial blood circulation $(n=1)$, facial size reduction and improvement of skin condition $(n=1)$, anti-aging by a patient-oriented self-assessment of facial elasticity scale $(n=1)$, and water and oil content of facial skin $(n=1)$. Four studies reported itching, dry desquamation, bruising, bleeding, pain, and swelling as adverse events. Six of 7 trials reported statistical flaws, and specific information regarding acupuncture rationale (42.9\%), other components of treatment (57.1\%), and practitioner background $(0 \%)$ were rarely reported. However, details regarding patient indications for treatment, treatment regimen, and control interventions were consistently reported (100\%) in the Standards for Reporting Interventions in Clinical Trials of Acupuncture (STRICTA) reporting guideline.

Conclusion: This systematic review suggests that a few trials regarding acupuncture for cosmetic use have incorporated rigorous designs. Although the studies reported generally positive results with tolerable safety, the methodology of the trials should be updated in a rigorous manner, including the use of randomized, sham-controlled studies with standardized interventions, in order to provide sufficient evidence regarding the effects of cosmetic acupuncture.
\end{abstract}

Keywords: acupuncture; cosmetic use; miso facial acupuncture; prospective study; systematic review

\section{Introduction}

In recent years, the popularity of cosmetic procedures has increased, concomitant with extended life expectancy. According to global statistics released by the International Society of
Aesthetic Plastic Surgery in 2017, there were 13,209,539 nonsurgical cosmetic procedures in 2016, approximately $10 \%$ more than the number performed in 2015. In both 2015 and 2016, the number of non-surgical procedures exceeded those of total surgical procedures. Interest in cosmetic acupuncture is similarly

Received October 11, 2018; Accepted November 26, 2018

Corresponding author: Byung-Cheul Shin

E-mail:drshinbc@pusan.ac.kr, ORCID: http://orcid.org/0000-0002-0059-2689

This is an Open Access article distributed under the terms of the Creative Commons Attribution Non-Commercial License (http://creativecommons.org/licenses/by-nc/4.0), which permits unrestricted non-commercial use, distribution, and reproduction in any medium, provided the original work is properly cited.

Copyright @ 2018 Korean Society of Korean Cosmetic Surgery and Medicine (KSKCS \& KCCS). 
increasing [1,2].

Cosmetic acupuncture is a type of cosmetic procedure, defined as the use of acupuncture on the head, face, and neck for cosmetic purposes without surgery and injection, such as to enhance facial muscle and skin tone, or for moisturizing purposes $[3,4]$. The primary aims of cosmetic acupuncture in Korea include facial wrinkle care, anti-aging, and reduction of facial size [5]. Though acupuncture has a long history, spreading from ancient China to Korea, Japan, and Western society in the early 1970s, acupuncture for cosmetic use is a recent concept resulting from the sharp increase in economic status at the end of the 20th century [4].

Despite the use of cosmetic acupuncture in clinical fields, there are few reports on the evidence of its effects and safety for cosmetic use $[4,5]$. There have been several reports regarding the use of cosmetic acupuncture for the rejuvenation of facial skin $[5,6]$ but these did not constitute systematic analyses of its effects or safety. Recently, an article was published analyzing facial cosmetic acupuncture in Korea [5], but it did not constitute a systematic review.

Previously published reviews did not adhere to Preferred Reporting Items for Systematic reviews and Meta-Analyses (PRISMA) reporting guidelines [7], and were not conducted systematically. Therefore, the aims of this study were to review the literature to determine the current status of the use of facial cosmetic acupuncture and to systematically evaluate the effects and safety of cosmetic acupuncture while adhering to the PRISMA reporting guidelines.

\section{Materials and methods}

\section{Data sources and searches}

The following electronic databases were searched up to August 2018. We searched 4 English databases (PubMed, Ovid LWW Medline, EMBASE, and Cochrane Library), 1 Chinese database (China National Knowledge Infrastructure [CNKI]), 1 Japanese database (J-stage), and 7 Korean databases (National Discovery for Science Leaders [NDSL], Korean Medical Database [KMBASE], Oriental Medicine Advanced Searching Integrated System [OASIS] and Korean Traditional Knowledge Portal [KTKP], Korean Studies Information Service System [KISS], Database Periodical Information Academic [DBpia], and the Korean National Assembly Digital Library [KNADL]).

The search terms used were as follows: ((acupuncture, cosmetic OR miso-acupuncture OR miso acupuncture) AND (prospective OR randomized OR randomized)). The search terms were slightly modified for acceptable use in each database. Furthermore, the references in searched articles were manually searched to identify further relevant studies.

\section{Study selection}

\section{Inclusion criteria}

This study included prospective trials that evaluated the effects of acupuncture on cosmetic conditions (e.g., acne vulgaris, facial wrinkles, and anti-aging). We included prospective trials that were either randomized controlled trials (RCTs), non-randomized controlled trials, or prospective, experimental, singlearmed studies.

Participants in the included studies underwent acupuncture to improve facial wrinkles, skin conditions, or with a broader goal of anti-aging; obese participants were excluded, regardless of gender, age, or ethnicity.

We included interventions using acupuncture needles inserted into the skin for cosmetic purposes only. Studies that assessed the combined effects of cosmetic acupuncture plus other interventions were also considered when the identical intervention was administered to both the cosmetic acupuncture group and the control group. If a single-armed study used cosmetic acupuncture with other interventions and cosmetic acupuncture was the primary intervention, we included the study after careful review. For control groups, we included any relevant active or sham controls.

We only included relevant outcomes with respect to cosmetic conditions, including total effective rate, wrinkle counts, and any other relevant outcomes measured using devices. Additionally, we included safety reports for the assessment of safety outcomes.

\section{Exclusion criteria}

We excluded case reports, non-systematic reviews, surveys, and case series. With respect to cosmetic acupuncture interventions, we excluded auricular acupuncture, embedding acupuncture, or needles attached solely to acupoints. Studies comparing different types of acupuncture were also excluded.

\section{Data extraction}

Two independent reviewers (B.C.S. and K.T.L.) screened the titles and abstracts of articles to identify potentially eligible studies and then examined the full texts to assess their final inclusion. Both authors cross-checked eligible studies; any disagreements regarding the extraction of study data were resolved 


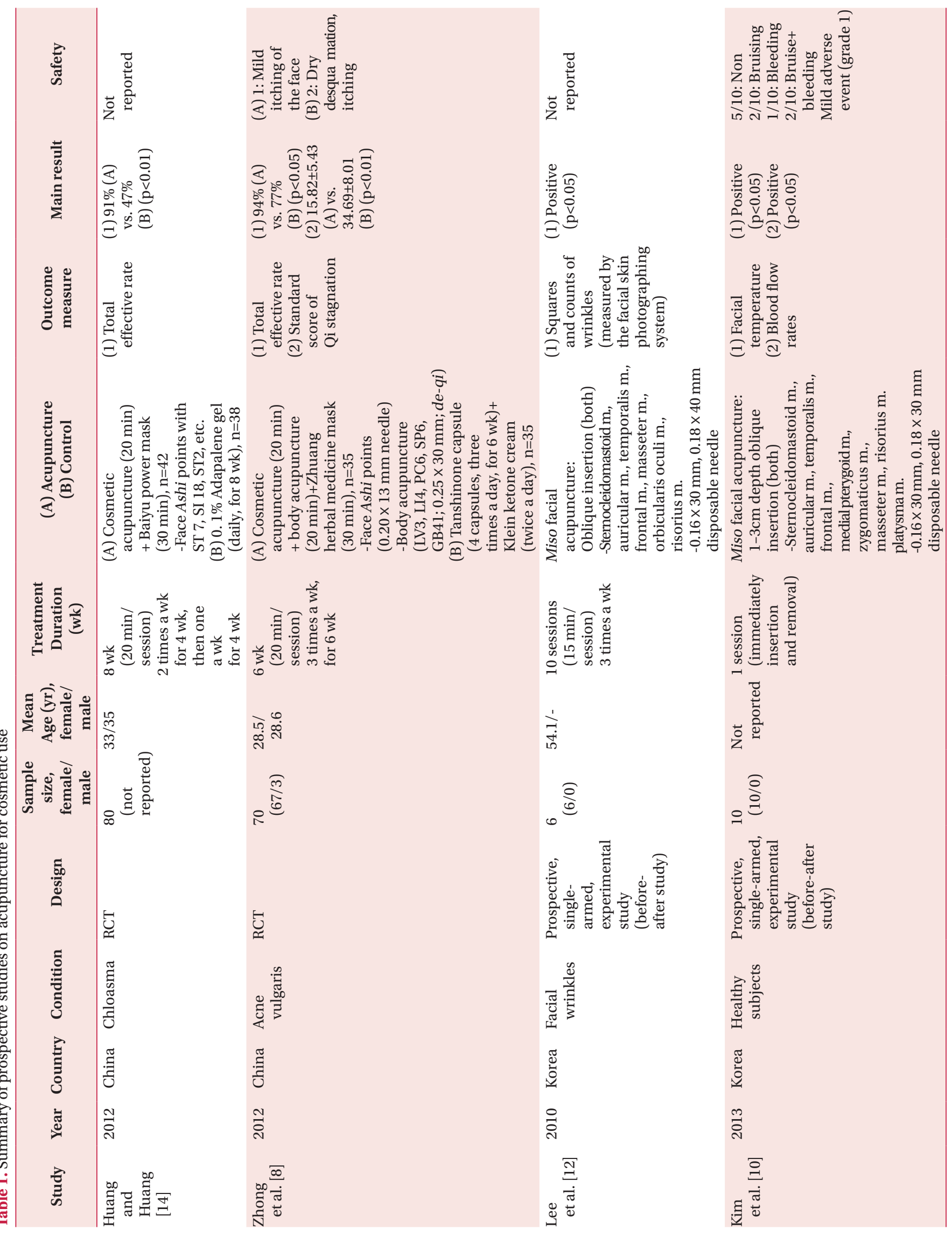




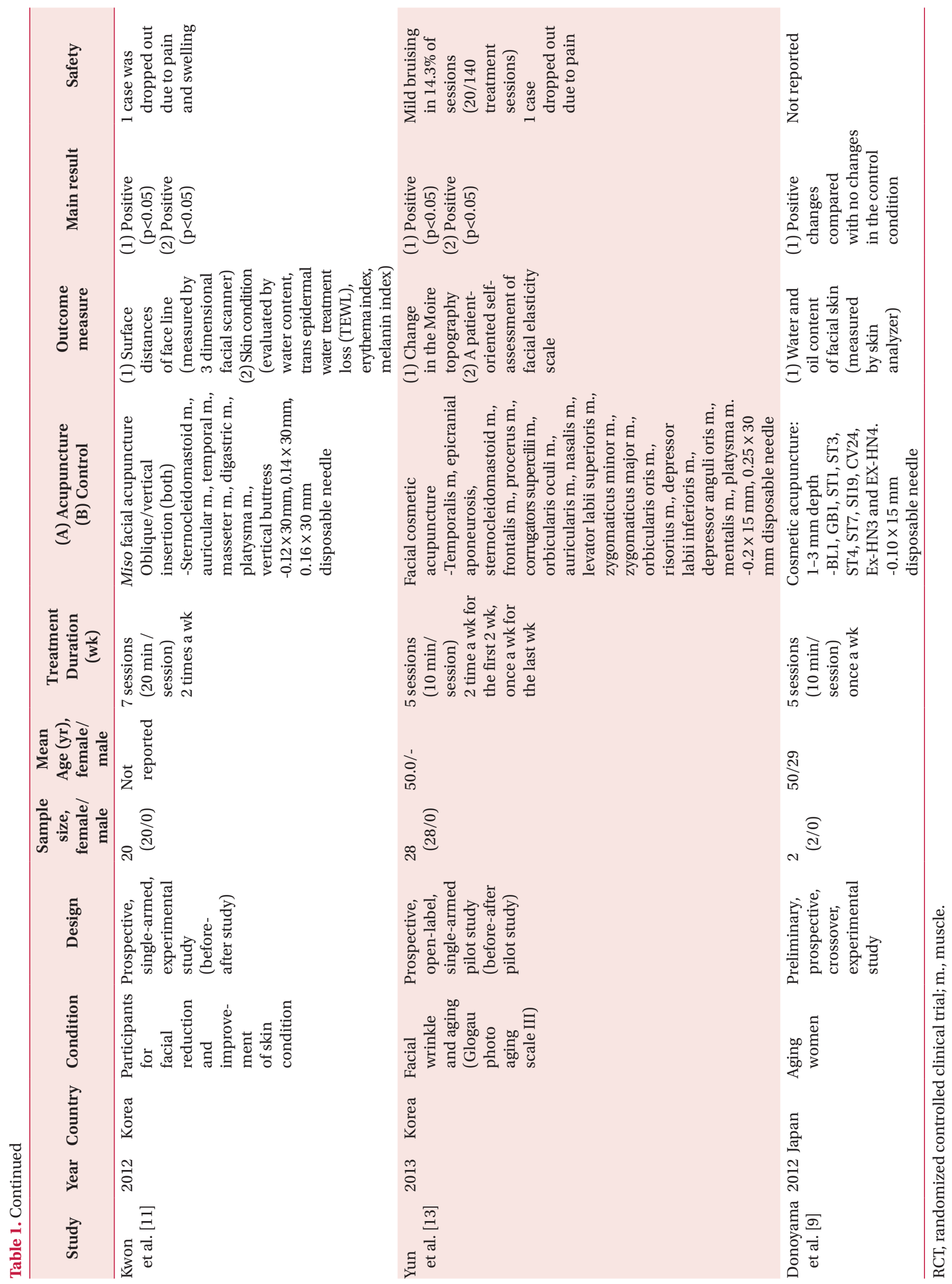




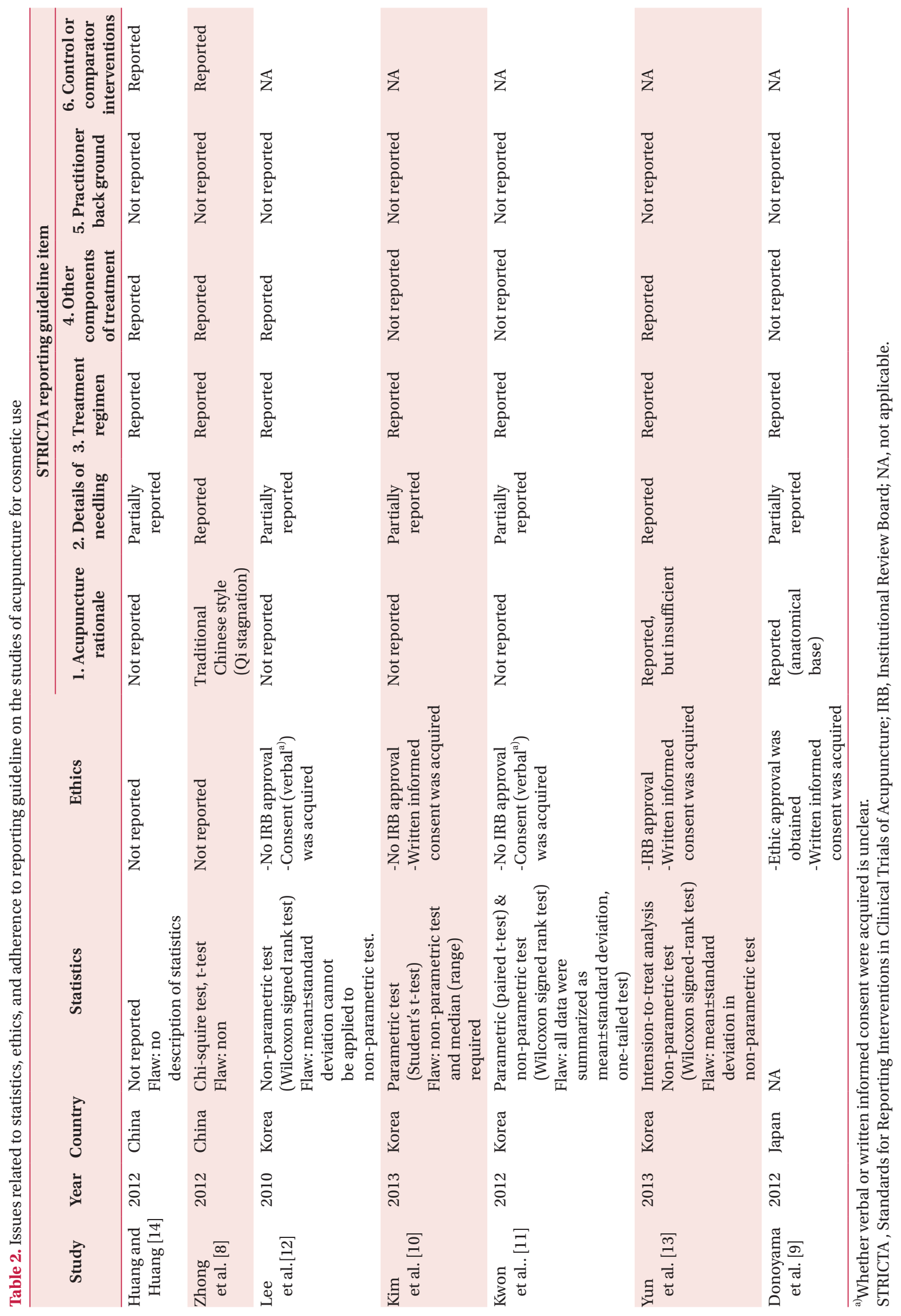


by discussion to reach consensus.

We extracted the following data from the articles selected for inclusion: authors, year of publication, types of cosmetic condition, study design, sample size, interventions, controls, duration of treatment, outcome measures, safety reports, and the main findings (Table 1) [8-14]. We also extracted data regarding statistical procedures, ethics, and adherence to the Standards for Reporting Interventions in Clinical Trials of Acupuncture (STRICTA) (Table 2) [8-15].

\section{Data analyses}

All data from outcome measurements were extracted, and we conducted recalculation with respect to effect size (ES) and power ( $\mathrm{G}^{*}$ power version 3.1.9.2 statistical software) between before and after data by comparing means of matched pairs or goodness-of-fit test by $\chi^{2}$ test ( $\sigma=0.05$, two-tailed). We used mean and standard deviation (or transformed), non-parametric data, or numnber (\%). Initially, we planned to conduct a meta- analysis. However, high heterogeneity prevented such metaanalysis because different conditions and outcome measures were used.

\section{Results}

\section{Study selection}

Our search yielded 283 records from English, Chinese, Japanese, and Korean databases, including 52 from the Cochrane library and PubMed, 152 from Korean databases, 61 from the Chinese database, and 18 from the Japanese database. After removal of duplicate studies, 157 records underwent screening of the title and abstract; 145 records were excluded (Fig. 1). The 12 remaining records were analyzed in greater depth to identify relevant studies; 5 were excluded in accordance with our exclusion criteria. Four RCTs were excluded because three trials compared cosmetic acupuncture to different acupuncture styles [16-18], while the other RCT comprised needling of acne

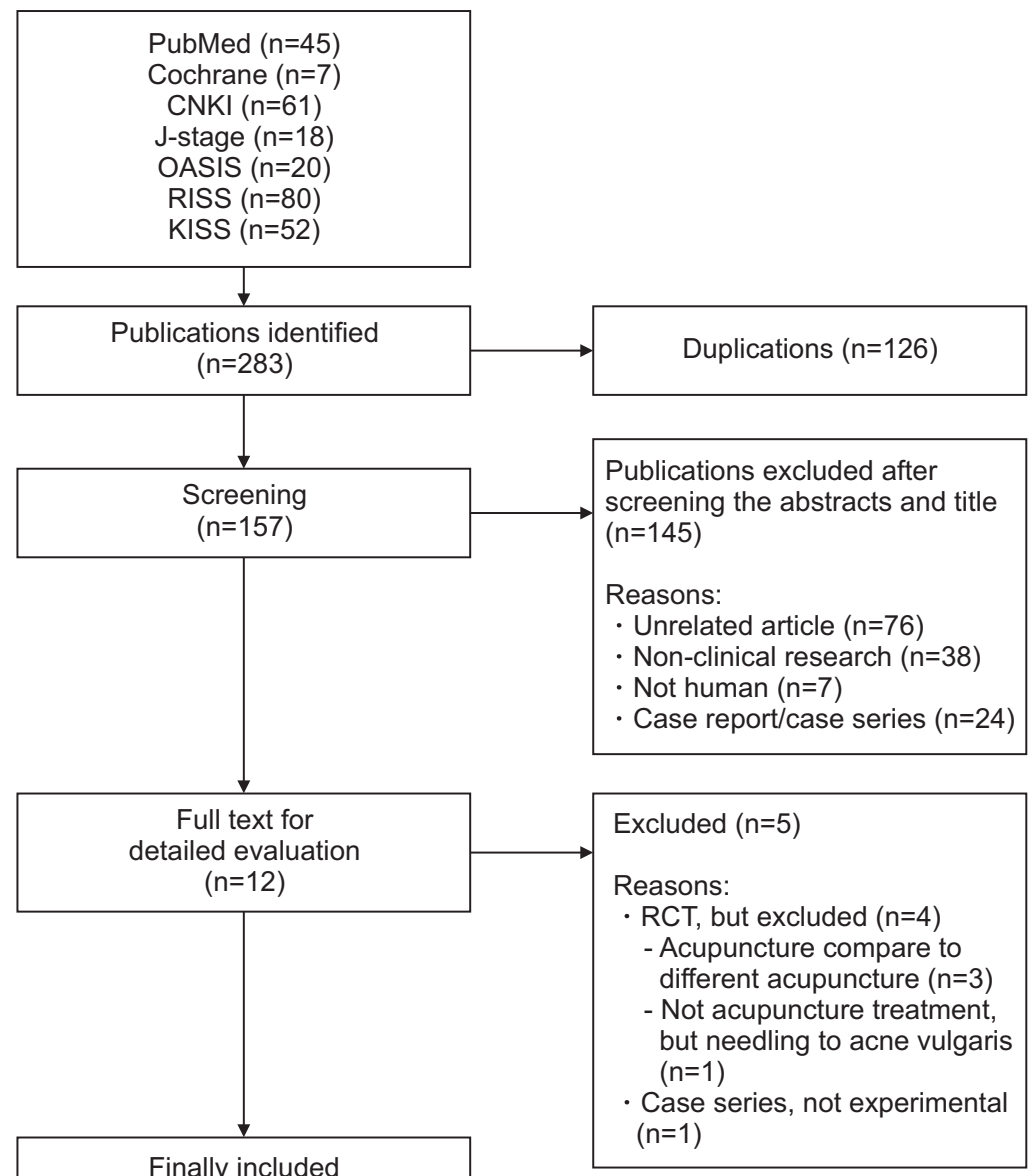

Fig. 1. PRISMA flow diagram of the search and selection process. RCT, randomized controlled trial. 
vulgaris [19]; the remaining study constituted a case series, rather than an experimental study [20]. All selection processes are summarized in Fig. 1, in accordance with PRISMA guidelines [7]. Finally, a total of 2 RCTs $[8,14]$ and 5 prospective, experimental studies [9-13] (Chinese, $n=2$; English, $n=2$; Korean, $n=3$ ) were included in our review. Detailed characteristics of the 7 studies included are summarized in Table 1.

\section{Study characteristics}

Two RCTs ( $\mathrm{n}=150)$ and 5 prospective, single-armed studies ( $n=66$ ) containing the data of 216 participants were included in the review. The number of participants in the 5 prospective, experimental studies ranged from 2 to 28 . All studies were from East Asia: 2 RCTs from China [8,14]; 4 prospective, experimental studies from Korea [10-13]; and 1 from Japan [9]. The number of total sessions was $8.3 \pm 5.6$ sessions (range, 1-18); the length of each session was $15.8 \pm 4.9$ minutes (range, 10-20).

The types of conditions were diverse and heterogeneous. The most common conditions treated by cosmetic acupuncture comprised facial care: facial wrinkles $(\mathrm{n}=2)[12,13]$, anti-aging $(\mathrm{n}=1)$ [9], facial size reduction and skin care $(\mathrm{n}=1)$ [11], acne vulgaris $(\mathrm{n}=1)$ [8], and chloasma $(\mathrm{n}=1)$ [14]; additionally, cosmetic acupuncture was used to perform changes in blood circulation of healthy subjects $(\mathrm{n}=1)[10]$ (Table 1$)$.

Outcome measures of the included studies were diverse and heterogeneous (Table 1).

\section{Effects and safety of facial cosmetic acupuncture}

All studies were diverse with respect to conditions and outcomes; therefore, we have reported the effects of included studies in a descriptive manner (Table 1).

\section{Effects of facial cosmetic acupuncture on wrinkle care}

Two prospective single-armed studies $[12,13]$ assessed the effects of cosmetic acupuncture on squares and counts of wrinkles, or on changes in Moire topography, in facial wrinkle care. Both reported positive effects after 5 or 10 sessions of cosmetic acupuncture treatment (Table 1).

\section{Effects offacial cosmetic acupuncture on skin conditions care}

Two prospective experimental studies $[9,11]$ assessed the effects of cosmetic acupuncture on water and oil content, erythema index, and melanin index for the improvement of skin condition. Both reported positive effects (Table 1).
Effects of facial cosmetic acupuncture on other facial conditions

One RCT [14] compared cosmetic acupuncture with $0.1 \%$ Adapalene gel for their effects on chloasma by assessing total effective rate; the results of that study were positive $(\mathrm{p}<0.05)$. The other RCT [8] tested the effects of cosmetic acupuncture on acne vulgaris, compared to Tanshinone capsule by measuring total effective rate. Its results were also positive $(\mathrm{p}<0.05)$.

A prospective study assessed the improvement of blood circulation after cosmetic acupuncture and reported positive results $(\mathrm{p}<0.05)$ (Table 1) [10].

\section{Safety reporting}

Only 4 of 7 studies $[8,10,11,13]$ reported on the safety of cosmetic acupuncture. Common adverse events were bruising, bleeding, pain, swelling, and itching. All were considered mild adverse events.

Evaluation of statistical methods, ethics, and adherence to acupuncture reporting guidelines

Six studies showed statistical flaws, one did not [8]. The primary shortcoming was the absence of a detailed description of statistical methods; moreover, non-parametric analyses were not used appropriately when the data were not normally distributed or exhibited a small sample size (Table 2).

Two studies reported institutional review board (IRB) approval $[9,13]$, while 5 studies reported obtaining consent from participants; 3 had written informed consent $[9,10,13]$ and 2 simply reported that consent $[11,12]$ had been obtained (Table 2).

Adherence to each item of the STRICTA reporting guidelines was as follows: 3/7 (42.9\%) for acupuncture rationale, 7/7 (100\%) for details of needling, $7 / 7(100 \%)$ for treatment regimen, 4/7 (57.1\%) for other components of treatment, 0/7 (0\%) for practitioner background, and $2 / 2(100 \%)$ for control or comparator interventions (Table 2).

\section{Recalculation of effect size and power}

Two RCTs showed ES=1.43, power=1.0 [14] and ES=0.72, power $=0.99$ [8], when recalculated by $\chi^{2}$ test.

Prospective experimental studies showed ES $=0.53$, power=0.19 with respect to counts of skin furrows [12]; ES=1, power $=0.80$ with respect to changes in facial temperature [10]; ES=1.87, power=1.0 with respect to water count [11]; and $\mathrm{ES}=0.60$, power $=0.86$ with respect to mean change in Moire topography [13]. One study could not be recalculated because 
minimal data were reported [9].

\section{Risk of bias of RCTs}

One RCT reported use of a random number table [8] but the other RCT did not report any use of randomization [14]. Blinding of practitioner, patients, and assessor was not reported in either RCT $[8,14]$.

\section{Discussion}

The purpose of our systematic review was to explore the current status of facial cosmetic acupuncture and to evaluate the effects and safety of facial acupuncture for cosmetic use. Our primary finding was that many studies used defective research methodology and exhibited poor adherence to the STRICTA reporting guidelines for clinical acupuncture trials.

Of the 2 RCTs from China, only 1 reported an adequate randomization method (random number table) [8]. Five prospective studies also exhibited a high risk of bias because they were not controlled studies [21]. No trial adopted a meaningful blinding method for practitioner, patients, and assessor. To explore the impact of cosmetic acupuncture with respect to any facial conditions, we conducted recalculation of ES and power of the included studies. However, 1 prospective study [12] did not exhibit full power (0.19); this indicated an underpowered study with potentially unreliable results. Future trials should include sufficient sample sizes; pre-enrollment sample size calculation is highly recommended. Another concern involves the research design of the included studies. Most included studies adopted heterogeneous definitions of wrinkles, skin care, anti-aging, acne vulgaris, and chloasma; thus, our review could not draw any meaningful conclusions or generate a combined conclusion from the synthesis of included studies.

An important finding is that treatment trends differ widely between China, Korea, and Japan. Generally, Korean acupuncture used Miso facial acupuncture for the cosmetic treatment of face and neck muscle, without using meridian or acupuncture theory [10-13]; Chinese and Japanese acupuncture comprised face Ashi points with meridian theory. Additionally, Chinese acupuncture used moderate needle length [8,14], whereas the Japanese style used shallow needling to the face (1-3 mm depth) [9].

Our analysis had several limitations. Although we found 5 Chinese RCTs, we excluded 3 of those [16-18] because they compared cosmetic acupuncture with different types of body acupuncture; thus, it was not possible to clearly elucidate acu- puncture effects. Another concern is the small sample size of the included studies. Our recalculations showed moderate ES and power; however, this may be a result of a biased, exaggerated effect [22]. All RCTs and prospective studies included in this study presented methodological weaknesses; this prevented us from making clear conclusions regarding the efficacy of cosmetic acupuncture in facial care.

One article reported extensive facial sclerosing lipogranulomatosis as a complication of cosmetic acupuncture [23]. However, overall adverse events of cosmetic acupuncture reported in our review appear tolerable.

Future trials should consider standardized cosmetic acupuncture intervention and comparison with acceptable controls, such as sham or active standard treatments with a standardized regimen. Additionally, researchers should adhere to STRICTA reporting guidelines with respect to acupuncture rationale (42.9\%), other components of treatment (57.1\%), and practitioner background (0\%) [15].

\section{Conclusion}

Our systematic review of 2 RCTs and 5 prospective, singlearmed studies demonstrated that there are a few trials with high-quality evidence regarding cosmetic acupuncture for facial cosmetic conditions. The safety of cosmetic acupuncture appears tolerable; however, well-designed high-quality studies, such as sham-controlled studies with standardized cosmetic acupuncture interventions, are needed to determine its effects on facial cosmetic conditions.

\section{Acknowledgments}

This study was supported by clinical research grant from $\mathrm{Pu}-$ san National University Hospital in 2018.

\section{Conflicts of interest}

The authors have nothing to disclose.

\section{References}

1. International Society of Aesthetic Plastic Surgery (ISAPS). ISAPS international survey on aesthetic/cosmetic procedures performed in 2016 [Internet]. Hanover (NH): ISAPS, 2014 [cited 2018 Aug 24]. Available from: https://www.isaps.org/blog/ isaps-statistics/. 
2. Maisel A, Waldman A, Furlan K, Weil A, Sacotte K, Lazaroff JM, et al. Self-reported patient motivations for seeking cosmetic procedures. JAMA Dermatol 2018;154:1167-74.

3. Barrett JB. Acupuncture and facial rejuvenation. Aesthet Surg J 2005;25:419-24.

4. Doran V. An introduction to facial revitalisation acupuncture. Eur J Orient Med 2007;5:4-9.

5. Kim TY. Trend analysis of facial cosmetic acupuncture study based on the Korean Traditional Medicine. J Acupunct Res 2013;30:125-37.

6. Fang Y, Fumihiko S, Mamoru U, Masahiro W, Akio T. Cosmetic acupuncture for Oriental Medicine treatment. J Osaka Dent Univ 2012;46:5-9.

7. Moher D, Liberati A, Tetzlaff J, Altman DG, The PRISMA Group. Preferred reporting items for systematic reviews and meta-analyses: the PRISMA statement. PLoS Med 2009;6:e1000097.

8. Zhong J, Fu LL, Fang G, Zhang XP, Lin C. Clinical study on the treatment of Qi stagnation type acne vulgaris with acupuncture combined with Zhuang herbal medicine mask. Nat Med Front China 2012;7:55-6.

9. Donoyama N, Kojima A, Suoh S, Ohkoshi N. Cosmetic acupuncture to enhance facial skin appearance: a preliminary study. Acupunct Med 2012;30:152-3.

10. Kim TY, Bak JP, Kim YM. Changes of facial temperature and blood flow rates by treatment of Miso facial rejuvenation acupuncture. Korean J Orient Physiol Pathol 2013;27:481-6.

11. Kwon GS, Kim JH, Lee KA, Lee SJ, Song JH, Song CH, et al. The effect of Miso facial acupuncture on facial reduction and improvement of skin condition. J Korean Acupunct Moxibustion Med Soc 2012;29:7-18.

12. Lee KM, Lim SC, Kim JS, Lee BH. A clinical study on facial wrinkles treated with Miso facial acupuncture-measured by the facial skin photographing system-. J Korean Acupunct Moxibustion 2010;27:101-7.

13. Yun Y, Kim S, Kim M, Kim K, Park JS, Choi I. Effect of facial cosmetic acupuncture on facial elasticity: an open-label, single-arm pilot study. Evid Based Complement Alternat Med 2013;2013:424313.

14. Huang YS, Huang W. Nursing experience of treatment of chloasma with cosmetic acupuncture combined with Biayu power mask. Mod J Integr Tradit Chin West Med 2012;21:2945-6.

15. MacPherson H, Altman DG, Hammerschlag R, Youping L, Taixiang W, White A, et al; STRICTA Revision Group. Revised STandards for Reporting Interventions in Clinical Trials of Acupuncture (STRICTA): extending the CONSORT statement. PLoS Med 2010;7:e1000261.

16. Chang C, Lyu Y, Cao Q, Zheng B, Wan Y, Xu J. Comparative study on repairing effect of lance needle and cosmetic needle for pustular acne. Acta Univ Tradit Med Sin Pharmacol Shanghai 2013;27:51-53, 57.

17. Chen J. [Clinical observation of acupuncture treatment for 68 Chloasma patients]. Shenzhen J Integr Tradit Chin West Med 2015;(14):61-2. Chinese.

18. Zhang J, Lu Y, Liu Q, Cao Q, Jiang Y, Ding J, et al. Therapeutic observation on lance needle for acne vulgaris. Shanghai J Acupunct Moxibustion 2013;(11):929-30.

19. Zeng X, Yu G. [75 Cases of acne vulgaris treated by staged nursing care]. Chin J Ethnomed Ethnopharm 2012;(16):106-7. Chinese.

20. Kwon NH, Kim CY, Shin YJ, Seo S, Song JH, Baek YH, et al. Clinical study on facial skin furrow measurement changes after Miso facial rejuvenation acupuncture. J Korean Acupunct Moxibustion Soc 2009;26:133-40.

21. Gluud LL. Bias in clinical intervention research. Am J Epidemiol 2006;163:493-501.

22. Button KS, Ioannidis JP, Mokrysz C, Nosek BA, Flint J, Robinson ES, et al. Power failure: why small sample size undermines the reliability of neuroscience. Nat Rev Neurosci 2013;14:365-76.

23. Bashey S, Lee DS, Kim G. Extensive facial sclerosing lipogranulomatosis as a complication of cosmetic acupuncture. Dermatol Surg 2015;41:513-6. 